\title{
The genome of a prasinoviruses-related freshwater virus reveals unusual diversity of phycodnaviruses
}

\author{
Hao Chen ${ }^{1}$, Weijia Zhang ${ }^{1,5}$, Xiefei Li ${ }^{1}$, Yingjie Pan ${ }^{1,3}$, Shuling Yan ${ }^{1,4}$ and Yongjie Wang ${ }^{1,2,3^{*}}$ (D)
}

\begin{abstract}
Background: Phycodnaviruses are widespread algae-infecting large dsDNA viruses and presently contain six genera: Chlorovirus, Prasinovirus, Prymnesiovirus, Phaeovirus, Coccolithovirus and Raphidovirus. The members in Prasinovirus are identified as marine viruses due to their marine algal hosts, while prasinovirus freshwater relatives remain rarely reported.

Results: Here we present the complete genomic sequence of a novel phycodnavirus, Dishui Lake Phycodnavirus 1 (DSLPV1), which was assembled from Dishui Lake metagenomic datasets. DSLPV1 harbors a linear genome of 181,035 bp in length ( $\mathrm{G}+\mathrm{C}$ content: 52.7\%), with 227 predicted genes and 2 tRNA encoding regions. Both comparative genomic and phylogenetic analyses indicate that the freshwater algal virus DSLPV1 is closely related to the members in Prasinovirus, a group of marine algae infecting viruses. In addition, a complete eukaryotic histone $\mathrm{H} 3$ variant was identified in the genome of DSLPV1, which is firstly detected in phycodnaviruses and contributes to understand the interaction between algal virus and its eukaryotic hosts.
\end{abstract}

Conclusion: It is in a freshwater ecosystem that a novel Prasinovirus-related viral complete genomic sequence is discovered, which sheds new light on the evolution and diversity of the algae infecting Phycodnaviridae.

Keywords: Phycodnaviridae, Prasinovirus, DSLPV1, Histone H3, Diversity

\section{Background}

The phycodnaviruses comprise genetically diverse, morphologically similar, large icosahedral $(100 \sim 200 \mathrm{~nm})$, and double strand DNA (180 $560 \mathrm{kbp})$ viruses that infect eukaryotic algae from both fresh and marine waters, and presently contain six genera: Chlorovirus, Prasinovirus, Prymnesiovirus, Phaeovirus, Coccolithovirus and Raphidovirus [1]. The family of Phycodnaviridae is placed within a major, monophyletic assemblage of large eukaryotic DNA viruses, termed the nucleo-cytoplasmic large DNA viruses (NCLDVs), which was recently proposed to group into a new viral order Megavirales $[2,3]$. Accumulating evidence suggests that these

\footnotetext{
*Correspondence: yjwang@shou.edu.cn

'College of Food Science and Technology, Shanghai Ocean University, Shanghai, China

2Laboratory for Marine Biology and Biotechnology, Qingdao National

Laboratory for Marine Science and Technology, Qingdao, China

Full list of author information is available at the end of the article
}

algae-infecting large viruses are active players in the aquatic ecosystem [4-6].

The hosts of phycodnaviruses are abundant and widespread in the natural environments, while almost all phycodnaviruses, including members of the Coccolithovirus, Phaeovirus, Prasinovirus, Prymnesiovirus and Raphidovirus genera, infect marine algae except for chloroviruses (Chlorovirus) that target freshwater algae $[7,8]$. The members of Prasinovirus are the most studied among these marine algae-infecting viruses because the hosts of prasinoviruses are worldwide distributed and play a central role in the oceanic carbon cycle [9]. All known prasinoviruses infect marine photosynthetic picoeukaryotic algae in the class Mamiellophyceae, which mainly contains three genera: Ostreococcus, Micromonas and Bathycoccus [10, 11]. Thus far, several prasinoviruses have been isolated and sequenced. For instance, the Ostreococcus virus OtV5 (Ostreococcus tauri virus 5), possessing 186,234 bp long linear genome, was isolated from the green alga $O$. tauri [12], and the Micromonas virus MpV1 (Micromonas sp. 
RCC1109 virus 1) was isolated from M. sp. RCC1109 in eutrophic northwestern Mediterranean coastal lagoons with a genome size of 184,095 bp [13]. Interestingly, in our previous work of culture-independent metagenomic analyses, genomes of prasinoviruses-related viruses, Yellowstone Lake Phycodnaviruses (YSLPVs), were discovered in Yellowstone Lake [14], a freshwater lake in Yellowstone National Park, Wyoming.

In this study, we assemble the complete genome of a Prasinovirus-related novel large phycodnavirus from freshwater metagenomic datasets in Shanghai, China. Comparative genomic and phylogenetic analyses reveal unique features of this new member of Phycodnaviridae and shed light on the evolution, diversity and distribution of phycodnaviruses.

\section{Methods}

\section{Metagenomic data source}

The Dishui Lake (DSL) metagenomic datasets were generated with Illumina Miseq sequencer and subjected to a series of processes for quality controlling as described previously [15]. In brief, water samples were taken from neritic area of Dishui Lake (121 $55^{\prime} 27.00^{\prime \prime}$ N 30 53'56.00' E) in 2013. Microbial biomasses were collected onto $0.22-\mu \mathrm{m}$ membrane filters, from which over $6 \mu$ g genomic DNA was extracted and used for metagenomic sequencing. Raw reads were firstly analyzed with FastQC and NGS QC Toolkit prior to de novo sequence assembly. Detail information of the datasets was shown in Additional file 1: Table S1.

\section{Metagenomic sequence assembly of viral genome}

All high-throughput sequenced reads in the DSL metagenomic paired-end libraries were assembled into contigs by using Newbler v2.6 (Roche) with default parameters. Among 1949 contigs with the length of more than $10 \mathrm{~kb}$, one was initially confirmed to be related to algal large viruses with BLASTP searching of NCBI nr database and subsequently used as the template in reference assembly as previously described [16]. Briefly, this contig was used as the reference sequence to which all reads in the DSL metagenomic paired-end datasets were assembled with a minimum overlap length of $25 \mathrm{bp}$ and minimum overlap identity of $95 \%$. The reference assembly was repeated until the assembled sequence stopped extending. All assemblies were performed with the bioinformatics software Geneious R9 (Biomatters, https://www.geneious.com).

\section{Genome analysis}

Geneious R9 software (Biomatters) was used to predict open reading frames (ORFs) by defining a start codon of ATG and a minimum 150 bp. Translated amino acid sequences were used to search $\left(\mathrm{E}\right.$-value $<10^{-3}$ ) for homologs in NCBI nr database by using the BLASTP program. One top hit to virus and/or non-virus was recorded. Functional annotation of ORFs was performed using the InterProScan program (http://www.ebi.ac.uk/ Tools/pfa/iprscan/) [17], and conserved domain was determined with both the NCBI server and the HHpred server (http://toolkit.tuebingen.mpg.de/hhpred) [18]. Transfer RNA (tRNA) sequences were identified by using the tRNAscan-SE tool [19]. Repetitive sequences were detected with both the Geneious Pro software and the Reputer program (http://bibiserv.techfak.uni-bielefeld.de/reputer/) [20]. Whole genome alignment at the nucleotide level was performed with the MAUVE software [21].

\section{Phylogenetic analysis}

Maximum likelihood phylogenetic trees were reconstructed based on homologs of DNA polymerase B family (PolB) gene, nicotinamide adenine dinucleotide (NAD)-dependent epimerase/dehydratase gene and histone $\mathrm{H} 3$ gene, respectively. Homolog counterparts from a range of representative viruses, bacteria and eukaryotes were downloaded from the NCBI protein database (https://www.ncbi.nlm.nih.gov/protein). Amino acid sequences were multiply aligned with the MUSCLE program, followed by tree reconstruction with the MEGA 7 software by using the JTT model and bootstrap value of 100 [22].

\section{PCR for the histone gene}

To avoid any possibilities of the misassembly of the histone gene in the assembled viral genome, a pair of PCR primers (5'-CTTGAGTATGGCACCCTTGG-3' and 5'-TCGCTTGGCGTCTTTCAAGG-3') respectively targeting the up- and downstream of the histone gene were designed based on the assembled vial genomic sequence. PCR was then performed with the same DNA samples as applied to the metagenomic sequencing. The reaction $(25 \mu \mathrm{L})$ contained $0.4 \mathrm{mM}$ of forward and reverse primers, $12.5 \mu \mathrm{L}$ of Taq PCR master mix $(2 \times)$ (Tiangen Biotech), and 20-25 ng of DNA. The amplification started with the program: initial denaturation at $94{ }^{\circ} \mathrm{C}$ for $4 \mathrm{~min}$, followed by 30 cycles of $94{ }^{\circ} \mathrm{C}$ for $30 \mathrm{~s}$, $58{ }^{\circ} \mathrm{C}$ for $30 \mathrm{~s}$, and $72{ }^{\circ} \mathrm{C}$ for $45 \mathrm{~s}$ in an Eppendorf Mastercycler machine. PCR products were cloned and sequenced as previously described [15]. Sequences were aligned to the assembled genome by using Geneious R9 (Biomatters, https://www.geneious.com).

\section{Homology modeling of Histone H3 in DSLPV1}

The three dimensional structure of histone fold $\mathrm{H} 3$ in DSLPV1_013 was modeled on the online protein homology modeling SWISS-MODEL server (www.swissmodel. expasy.org) with the template of the crystal structure of human histone H3 (PDB id 3lel.1.E, amino acid sequence identity 83.82\%). The structure was visualized and analyzed with the PyMOL software (www.pymol.org) [23]. 
NCLDV conserved genes and virophage related genes in DSLPV1

The nucleo-cytoplasmic virus orthologous group proteins (NCVOG) [24] were downloaded from NCBI (ftp://ftp.ncbi.nih.gov/pub/wolf/COGs/NCVOG) for establishing a local database by using 'make blastdb' command in a BioLinux server. Similarly, all predicted proteins of Dishui Lake Virophage 1 (DSLV1, accession number KT894027) were downloaded to construct another local dataset. Predicted proteins of DSLPV1 were used as queries to search these two datasets respectively by using BLASTP program with cutoff e-value of 1e-3. The output results were recorded and analyzed for the NCLDV conserved genes and virophage related genes in DSLPV1.

\section{Nucleotide sequence accession number}

The DSLPV1 sequence has been deposited in the GenBank database (accession no. KY747489).

\section{Results}

\section{General organization of the DSLPV1 genome}

After the reference assembly, more than 8000 reads were eventually mapped to the consensus sequence (the contig that was initially confirmed to be related to algal large viruses, which is described in "Metagenomic sequence assembly of viral genome" in Methods.) with a high coverage (average coverage $=78.7$, Additional file 1: Table S2) across the whole genome (Additional file 1: Figure S1), which indicated the accuracy of sequence assembly. The obtained genomic sequence with a size of 181,035 bp was named that of Dishui Lake Phycodnavirus 1 (DSLPV1). A 402-bp terminal inverted repeat was identified at both ends of the genome (Fig. 1a), which indicates the complete and linear genome of the DSLPV1. The G+C content of the genome is $52.7 \%$, which is much higher than that of prasinoviruses (37.0-44.6\%) but resembles YSLPVs (47.7-55.0\%). A total of 227 ORFs were predicted, which are evenly distributed on both positive and negative DNA strands through the genome (i.e. $48 \%$ on the positive strand) with an average gene length of $770 \mathrm{bp}$ and a coding density of 1.254 genes per kbp.

As the results of BLASTP search against NCBI nr database showed (Additional file 1: Table S3), among these 227 ORFs, 167 ( 74\%) shared similarity with proteins in $\mathrm{nr}$ database, with 155 having the top hits to algae large/giant viruses in NCLDVs, 6 to bacteria, 4 to eukaryotes and 2 to archaea (Fig. 1 and Additional file 1: Table S3). Among the remaining 60 ORFs ( 26\%) having no hits in NCBI nr database (Fig. 1a), 35 had hits to NCBI environmental database (env_nr). Interestingly, 98\% (152 of 155) ORFs having the best matches to giant/large viruses were homologous to algal large viruses in the Prasinovirus genus of the Phycodnaviridae family (Fig. 1b). Meanwhile, 75 out of these 155 ORFs with virus-hits showed no BLAST hits to cellular life (Additional file 1: Table S3), which are considered as virus specific genes, and the rest of 80 ORFs had both virus and non-virus hits (Additional file 1: Table S3). Of the four eukaryote-derived ORFs, one showed the best match to Ostreococcus lucimarinus CCE9901, the natural host of prasinoviruses, which indicates the potential genetic links between algae host and DSLPV1. In addition, two regions encoding tRNA (one Gln-tRNA and one Asn-tRNA) were identified in the DSLPV1 genome.

\section{Gene annotation in the DSLPV1 genome}

Functional annotation analysis of 227 putative proteins in DSLPV1 indicated that 160 (70.5\%) had no homologues with defined functions in public databases. The remaining 67 putative proteins (29.5\%) with annotated functions were classified into nine functional categories (Table 1). Ten putative proteins are involved in DNA replication, recombination and repair, which imply the independence of viral DNA replication in host cell; three putative proteins are associated with nucleotide transport and metabolism, eight with transcription, eight with sugar manipulation, three with DNA restriction/methylation, and eight with protein and lipid synthesis/modification; seven encode capsid proteins; three have other miscellaneous functions, and one possibly possesses a signaling function.

\section{NCLDV conserved genes in the DSLPV1 genome}

DSLPV1 contains a nearly complete set of conserved genes in NCLDVs (40/47), including core genes of DNA polymerase B family protein, A18 helicase, A32 virion packaging ATPase and seven copies of major capsid protein. Ten NCVOGs in prasinoviruses were present in DSLPV1, and other key feature genes in prasinoviruses were also detected in DSLPV1 (Table 2). Notably, although some core genes were undetected in DSLVP1, e.g., serine/threonine protein kinase, they were probably functionally displaced by other genes. For instance, DSLVP1 ORF27 has a protein kinase structure which could be the functional substitute of the serine/threonine protein kinase. Such varied genomic features distinguish DSLPV1 from the prasinoviruses.

\section{Genomic architecture of DSLPV1}

Whole genome of DSLPV1 was aligned with that of the prasinoviruses (OtV5, MpV1, BpV2) and YSLPV1-3, respectively (Fig. 2a). DSLPV1 revealed good sequence synteny with the prasinoviruses, but shared few homologous segments with the YSLPVs. In addition, the gene content analysis also showed that DSLPV1 shared more homologous genes with these three prasinoviruses (OtV5: 59\%, MpV1: 58\%, BpV2: 61\%) than with YSLPV1 (42\%). These results suggest the DSLPV1 genomic architecture is more analogous to the prasinoviruses than to the YSLPVs. 


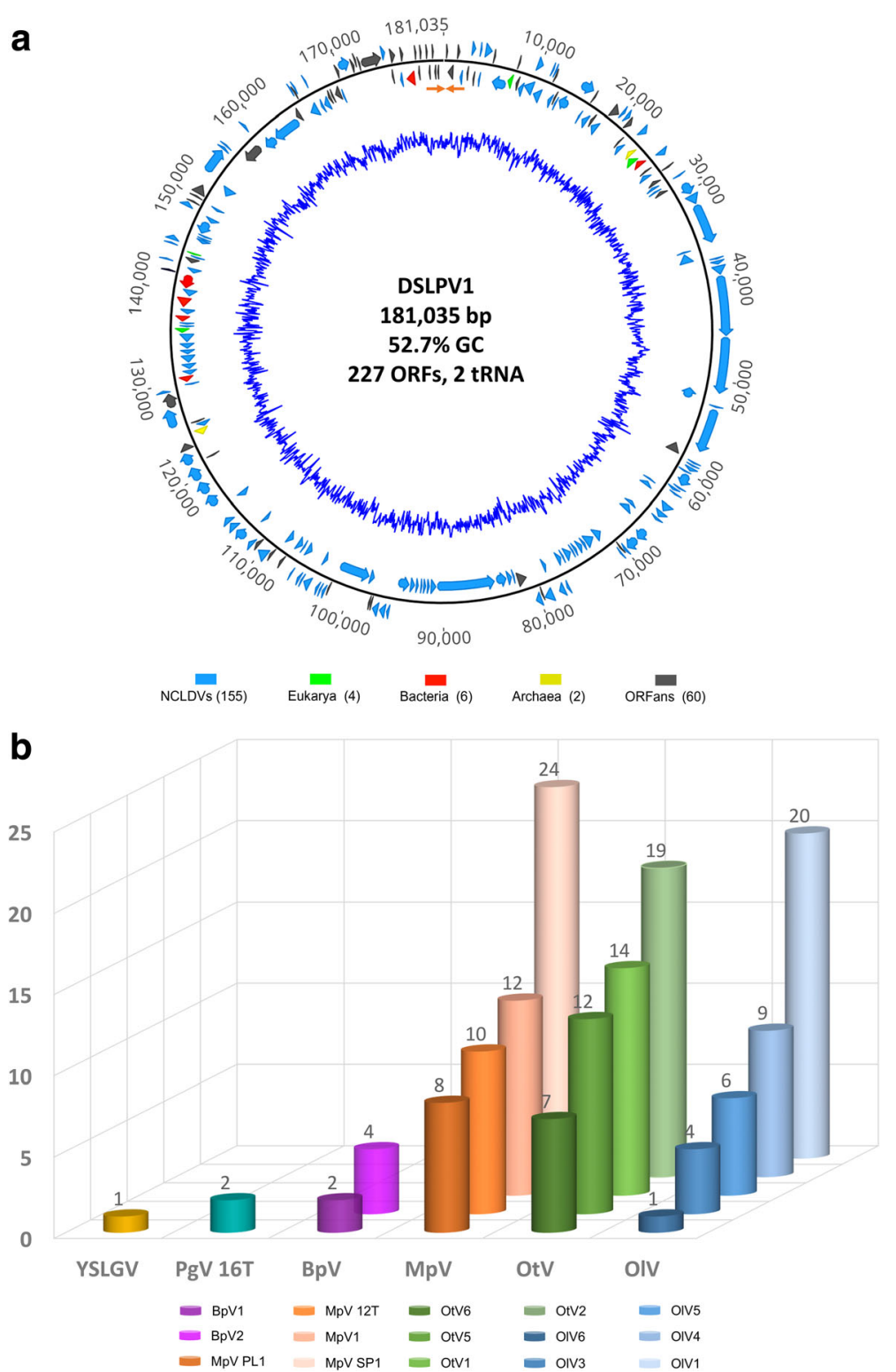

Fig. 1 Features of the DSLPV1 genome. a Genomic map of DSLPV1. The linear genome of DSLPV1 is shown in an open circle. The terminal inverted repeats of the linear genome are indicated with orange arrows. The outside numbers represent the position of nucleotide. ORFs are indicated with box arrows in different colors, which represent different taxon categories of the ORFs (forward and reverse strands respectively): light blue for NCLDVs hits ( $n=155)$, light green for eukaryote hits $(n=4)$, red for bacteria hits $(n=6)$, yellow for archaea hits $(n=2)$ and gray for ORFans (ORFs with no BLAST hits in public databases, $n=60$ ). The inner blue line represents $G+C$ content. Viral name, genomic length, $G+C$ content and the total number of predicted ORFs and the number of tRNA are showed in the center. $\mathbf{b}$ The number of top BLASTP hits of the DSLPV1 ORFs to large/giant viruses that infect algae. Different species are indicated in different colors. The full name of each virus is shown as following: BpV- Bathycoccus sp. RCC1105 virus; MpV- Micromonas pusilla virus; OIV- Ostreococcus lucimarinus virus; OtV- Ostreococcus tauri virus; PgV 16 T- Phaeocystis globosa virus 16 T; YSLGV- Yellowstone lake giant virus

\section{Phylogenetic affiliation of DSLPV1}

The annotated DNA polymerase gene (DSLPV1_189) of DSLPV1 shared high amino acid similarity (52-60\%) with that of other phycodnaviruses, especially the prasinoviruses and the YSLPVs. Phylogenetic analysis of this gene and its homologues in other giant/large viruses indicated that DSLPV1 formed a monophyletic group with
Prasinovirus with robust bootstrap support, and both DSLPV1 and the prasinoviruses are the sister lineages to the YSLPVs (Fig. 2b).

\section{Host like genes in the DSLPV1 genome}

A total of 44 ORFs in the DSLPV1 genome showed amino acid similarity (coverage $>70 \%$, identity $>25 \%$ ) to 
Table 1 DSLPV1 ORFs with annotated functions

\begin{tabular}{|c|c|}
\hline Function and protein & ORF \\
\hline \multicolumn{2}{|l|}{ DNA, RNA, replication, recombination and repair } \\
\hline DNA polymerase & 189 \\
\hline DNA topoisomerase I & 133 \\
\hline DNA topoisomerase $\|$ & 195 \\
\hline PCNA & 96 \\
\hline Exonuclease & 115 \\
\hline RNase $\mathrm{H}$ & 142 \\
\hline SF3 helicase & 152 \\
\hline NTPase/helicase & 104 \\
\hline W A18 helicase & 52 \\
\hline DNA polymerase III epsilon subunit & 209 \\
\hline \multicolumn{2}{|l|}{ Nucleotide transport and metabolism } \\
\hline Ribonucleotide reductase, large subunit & 119 \\
\hline Ribonucleotide reductase, small subunit & 139 \\
\hline ATPase (W A32 virion packaging ATPase) & 80 \\
\hline \multicolumn{2}{|l|}{ Transcription } \\
\hline mRNA capping enzyme & 77 \\
\hline RNase III & 114 \\
\hline TATA-box binding protein & 125 \\
\hline Poxvirus late transcription factor VLTF3 & 98 \\
\hline Nudix hydrolase & 68 \\
\hline Transcription elongation factor S-II & 178 \\
\hline Transcription factor MYB1R1 & 163 \\
\hline Transcription initiation factor IIB & 162 \\
\hline \multicolumn{2}{|l|}{ Sugar manipulation enzymes } \\
\hline Mannosyltransferase & 170 \\
\hline $\mathrm{N}$-acetylglucosamine transferase & 42 \\
\hline Glycosyltransferase & 43 \\
\hline Glycosyltransferase & 62 \\
\hline Glycosyltransferase & 166 \\
\hline Glycosyltransferase & 169 \\
\hline Glycosyltransferase & 200 \\
\hline NAD-dependent epimerase/dehydratase & 41 \\
\hline \multicolumn{2}{|l|}{ DNA restriction/methylation } \\
\hline Adenine-specific methyltransferase & 10 \\
\hline DNA-cytosine methyltransferase & 149 \\
\hline Methyltransferase FkbM & 100 \\
\hline \multicolumn{2}{|l|}{ Protein and lipid synthesis/modification } \\
\hline Prolyl 4-hydroxylase alpha-subunit & 103 \\
\hline Ubiquitin C-terminal hydrolase & 158 \\
\hline ATP-dependent protease proteolytic subunit & 16 \\
\hline FtsH2 metalloprotease & 12 \\
\hline $33 \mathrm{kDa}$ in vitro translation peptide & 118 \\
\hline $\mathrm{N}$-myristoyltransferase & 76 \\
\hline
\end{tabular}

Table 1 DSLPV1 ORFs with annotated functions (Continued)

\begin{tabular}{ll}
\hline Function and protein & ORF \\
\hline Patatin phospholipase & 106 \\
Asparagine synthetase & 181 \\
Capsid & 53 \\
Capsid protein 1 & 59 \\
Capsid protein 2 & 81 \\
Capsid protein 3 & 82 \\
Capsid protein 4 & 147 \\
Capsid protein 5 & 188 \\
Capsid protein 6 & 194 \\
Capsid protein 7 & \\
Miscellaneous & 105 \\
Virus inclusion body & 217 \\
Rhodanese domain-containing protein & 8 \\
Fibronectin-binding protein & \\
Signaling & 183 \\
PhoH
\end{tabular}

ORF numbers refer to that of DSLPV1. Their GenBank annotations are shown in Additional file 1: Table S3

genes found in the algae from both freshwater and marine environments, especially 12 ORFs that share high amino acid similarity (30-87\%) to genes in the Mamiellophyceae class (green algae), containing natural hosts of the prasinoviruses (Table 3). Meanwhile, DSLPV1_139, the putative ribonucleoside-diphosphate reductase small subunit gene (rnr2) that is often shared by the prasinoviruses and their algae hosts, showed high amino acid similarity (63\%) to the rnr2 in Ostreococcus lucimarinus CCE9901 (Table 3). In addition, DSLPV1 041 encodes a putative NAD-dependent epimerase/dehydratase protein that appears to derive from Ostreococcus lucimarinus CCE9901 as well (Table 3, Additional file 1: Figure S2).

\section{Complete eukaryotic histone fold $\mathrm{H} 3$ in the DSLPV1 genome}

Interestingly, DSLPV1 harbored a histone like gene (DSLPV1_013), which had high amino acid similarity (74-88\%) to the histone gene from a wide range of eukaryotes. To eliminate the possibility of metagenomic sequence misassembly, the histone gene of DSLPV1_013 was re-checked with the DSLPV1 specific PCR and sequencing analysis. Sequence alignment analysis revealed that mismatches of the histone gene were not observed between the PCR amplified sequence and the assembled genome, which confirms the presence of a histone like gene in DSLPV1. Protein fold recognition with the InterProScan software detected a complete histone fold $\mathrm{H} 3$ at the $\mathrm{C}$ terminal of this protein (Fig. 3a). Moreover, sequence comparison of representative histone $\mathrm{H} 3$ folds 
Table 2 Conserved genes present in DSLPV1 and the prasinoviruses

\begin{tabular}{|c|c|c|c|c|c|c|}
\hline & Gene name & Otv5 & MpV1 & BpV1 & $\mathrm{BpV} 2$ & DSLPV1 \\
\hline \multirow[t]{5}{*}{ NCLDV core gene } & DNA polymerase & 1 & 1 & 1 & 1 & 1 \\
\hline & ATPase (DNA packaging) & 1 & 1 & 1 & 1 & 1 \\
\hline & A18 helicase & 1 & 1 & 1 & 1 & 1 \\
\hline & Capsid protein & 8 & 8 & 7 & 7 & 7 \\
\hline & Serine/Threonine protein kinase & 1 & 1 & 1 & 1 & 0 \\
\hline \multirow[t]{14}{*}{ NCVOGs present in prasinoviruses } & Poxvirus late transcription factor VLTF3 & 1 & 1 & 1 & 1 & 1 \\
\hline & DNA topoisomerase & 2 & 1 & 1 & 1 & 2 \\
\hline & Transcription factor IIS & 1 & 1 & 1 & 1 & 1 \\
\hline & Transcription factor IIB & 1 & 1 & 1 & 1 & 1 \\
\hline & Ribonucleotide reductase small subunit & 1 & 1 & 1 & 1 & 1 \\
\hline & Ribonucleotide diphosphate reductase large subunit & 1 & 1 & 1 & 1 & 1 \\
\hline & mRNA capping enzyme & 2 & 2 & 2 & 2 & 2 \\
\hline & ATP dependant DNA ligase & 1 & 1 & 1 & 1 & 0 \\
\hline & Exonuclease (YqaJ) & 2 & 2 & 2 & 2 & 1 \\
\hline & dUTP pyrophosphatase & 1 & 1 & 1 & 1 & 0 \\
\hline & Thymidine kinase & 1 & 1 & 1 & 1 & 0 \\
\hline & Thymidylate S & 1 & 1 & 1 & 1 & 0 \\
\hline & Nudix hydrolase & 1 & 1 & 1 & 0 & 1 \\
\hline & $\begin{array}{l}\text { RuvC, Holliday junction resolvases, Extended Pox_A22, } \\
\text { Poxvirus A22 family }\end{array}$ & 2 & 2 & 1 & 1 & 2 \\
\hline \multirow[t]{18}{*}{ Other prasinovirus common genes } & PCNA & 1 & 1 & 1 & 1 & 1 \\
\hline & NTPase/helicase & 1 & 1 & 1 & 1 & 1 \\
\hline & RNase $\mathrm{H}$ & 1 & 1 & 1 & 1 & 1 \\
\hline & RNase III & 1 & 1 & 1 & 1 & 1 \\
\hline & Transcription activator /SWI/SNF helicase & 1 & 1 & 1 & 1 & 0 \\
\hline & TATA-box binding protein (TBP) & 1 & 1 & 1 & 1 & 1 \\
\hline & Prolyl 4-hydroxylase & 1 & 1 & 1 & 1 & 1 \\
\hline & $33 \mathrm{kDa}$ in vitro translation peptide & 2 & 3 & 2 & 2 & 2 \\
\hline & 2OG-Fe(II) oxygenase & 1 & 1 & 1 & 1 & 1 \\
\hline & Aspartyl/Asparaginyl beta-hydrolase & 1 & 1 & 1 & 1 & 0 \\
\hline & Ubiquitin hydrolase-like cystein peptidase & 1 & 1 & 1 & 1 & 1 \\
\hline & FtsH (3-4-24) metalloendopeptidase & 1 & 1 & 1 & 1 & 1 \\
\hline & ATP-dependant protease proteolytic subunit & 1 & 1 & 1 & 1 & 1 \\
\hline & Patatin-like phospholipase & 1 & 1 & 1 & 1 & 1 \\
\hline & Potassium channel protein, $\mathrm{PhoH}$ & 1 & 1 & 1 & 1 & 1 \\
\hline & $A B C-1$ domain protein & 1 & 1 & 1 & 1 & 1 \\
\hline & ATP/GTP binding site motif A AGB-1 & 1 & 1 & 1 & 1 & 1 \\
\hline & Viral A-type inclusion protein & 1 & 1 & 1 & 1 & 1 \\
\hline
\end{tabular}

The numbers in the table represent that of gene copies

from eukaryotes, selected based on BLASTP results, with that of DSLPV1 revealed high conservation between them (Fig. 3b), and the phylogenetic analysis indicates that the histone gene of DSLPV1 is closely related to that of eukaryotes but not viruses (Fig. 3c).
Meanwhile, the predicted structure of DSLPV1 histone fold $\mathrm{H} 3$ revealed a canonical eukaryotic histone fold (Fig. 3d). In addition, the $\mathrm{G}+\mathrm{C}$ content of the DSLPV1 histone gene $(57 \%)$ is incongruous with that of the DSLPV1 genome (52.7\%) but coherent with that of some 
a

DSLVP1 vs Prasinovirus

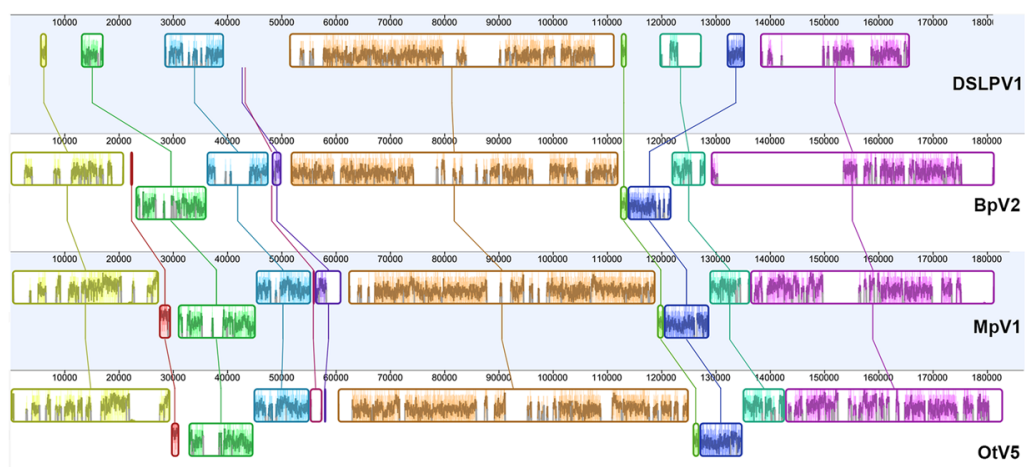

\section{DSLVP1 vs YSLPVs}

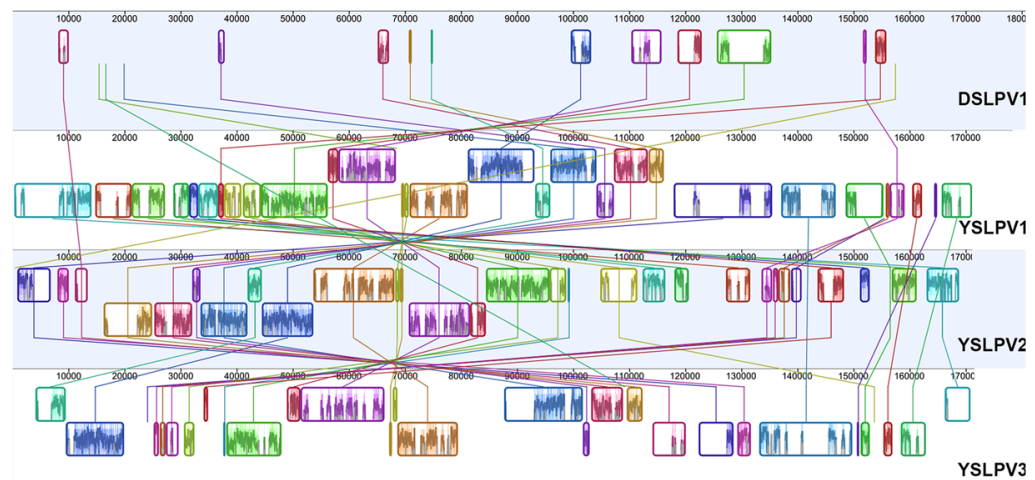

b

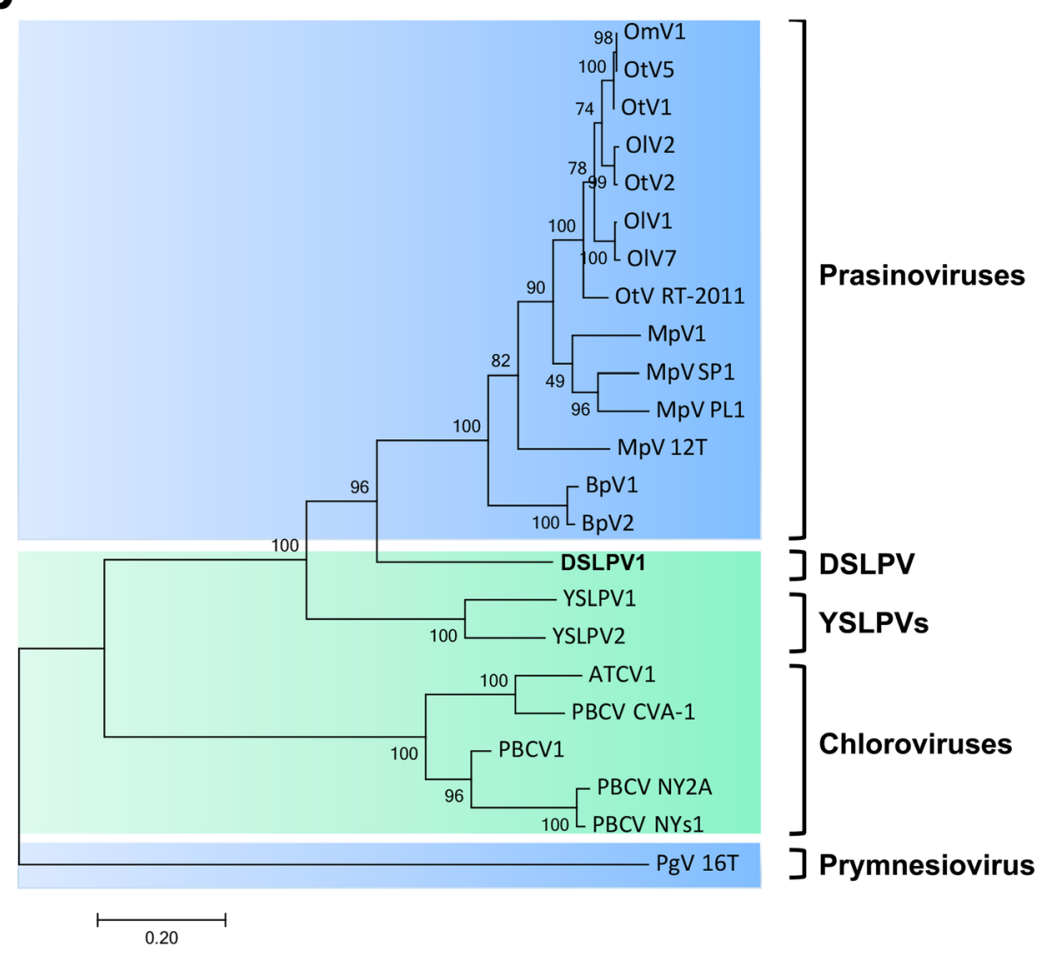

Fig. 2 (See legend on next page.) 
(See figure on previous page.)

Fig. 2 Relationship of DSLPV1 to the phycodnaviruses. a Whole genome alignment of DSLPV1 with the representative prasinoviruses and the YSLPVs. Different conserved regions shared among the genomes are indicated in different colors. b Maximum-likelihood phylogenetic tree of the B-family DNA polymerase (PolB) proteins. Marine viruses are shadowed in light blue, and freshwater viruses are shadowed in light green. Different families/groups of viruses are labeled with black lines and names on the right of the tree. The scale bar indicates a distance of 0.2 fixed mutations per amino acid position. GenBank accession number of the PolB sequences used for this tree is listed in Additional file 1: Table S4. OmV: Ostreococcus mediterraneus virus; Otv: Ostreococcus tauri virus; OIV: Ostreococcus lucimarinus virus; MpV: Micromonas pusilla virus; BpV: Bathycoccus sp. RCC1105 virus; DSLPV: Dishui Lake Phycodnavirus; YSLPV: Yellowstone Lake phycodnavirus; ATCV: Acanthocystis turfacea Chlorella virus; PBCV: Paramecium bursaria Chlorella virus; PgV: Phaeocystis globosa virus

algal genomes $[25,26]$. Taken together, these results indicate the possibility of a recent horizontal transfer of the histone gene from eukaryotic hosts, e.g., algae, to viruses.

\section{Genetic links between DSLPV1 and DSLV1}

Intriguingly, three homologous genes that are shared between DSLPV1 and Dishui Lake Virophage 1 (DSLV1) were detected based on BLAST when searching the local database that consists of all predicted DSLV1 proteins by using the baits of 227 ORFs of DSLPV1. Functional annotation of these three homologous proteins revealed that the DSLV1_02 harbored two transmembrane regions at N terminal, which were analogous to DSLPV1_019 (26.6\% of amino acid similarity) and DSLPV1_141 (25.4\% of amino acid similarity). Similar RING-type zinc finger domain was detected at C terminal of DSLV1_25 and DSLPV1_175 (29.4\% of amino acid similarity), and this domain is probably involved in mediating protein-protein interactions. In addition, a collagen triple helix repeat domain at $\mathrm{C}$ terminal of DSLV1_09 showed 41.2\% of amino acid identity to DSLPV1_201, which represents the highest sequence similarity in comparison with the similarity that was shared between the other homologies described above.

\section{Discussion}

In this study, the genomic sequence of a novel phycodnavirus, named DSLPV1, was obtained from the DSL metagenomic datasets. The DSLPV1 genome appears to be complete as it contains a pair of 402-bp terminal inverted repeats flanking both ends of the linear genome as well as a nearly complete set of conserved genes of NCVOGs. Both comparative genomic and phylogenetic analyses indicate that DSLPV1 is closely affiliated to the prasinoviruses since they are similar in genomic length, share the most number of homologous genes including almost complete set of the Prasinovirus conserved genes (27/32), and formed a monophyletic clade based on the DNA PolB protein phylogeny. Accordingly, DSLPV1 currently represents the closest freshwater relative of the marine prasinoviruses.

Dishui Lake where DSLPV1 was discovered is a holomictic freshwater lake $(0.8 \%$ of salt) that was artificially excavated on tidal flat in 2003. Freshwater from neighboring Dazhi River is diverted into Dishui Lake, which is regularly discharged to the East China Sea. Interestingly, DSLPV1 is closely affiliated with the Prasinovirus infecting marine algae in the Mamiellophyceae class rather than with the freshwater-originated Prasinovirus-related

Table 3 Host like genes in DSLPV1

\begin{tabular}{|c|c|c|c|c|c|}
\hline ORF & Putative function & Hit & Identity (\%) & Coverage (\%) & E value \\
\hline DSLPV1_008 & Fibronectin-binding protein & $\mathrm{OL}$ & 38 & 95 & $8.00 \mathrm{E}-11$ \\
\hline DSLPV1_013 & Histone $\mathrm{H} 3$ & $\mathrm{OL}$ & 87 & 71 & 1.00E-78 \\
\hline DSLPV1_041 & NAD-dependent epimerase & $\mathrm{OL}$ & 48 & 97 & $3.00 \mathrm{E}-89$ \\
\hline DSLPV1_043 & Glycosyltransferase & OT & 27 & 69 & 5.00E-09 \\
\hline DSLPV1_076 & $\mathrm{N}$-myristoyltransferase & MP & 36 & 99 & $1.00 \mathrm{E}-48$ \\
\hline DSLPV1_096 & PCNA & MP & 30 & 97 & $3.00 \mathrm{E}-31$ \\
\hline DSLPV1_113 & DNA adenine methyltransferase & Ms & 33 & 93 & 2.00E-08 \\
\hline DSLPV1_139 & Ribonucleotide reductase, ss & $\mathrm{OL}$ & 63 & 73 & $2.00 E-135$ \\
\hline DSLPV1_159 & mRNA-capping enzyme & OT & 26 & 93 & $5.00 \mathrm{E}-24$ \\
\hline DSLPV1_164 & Unknown & MP & 46 & 96 & $3.00 \mathrm{E}-18$ \\
\hline DSLPV1_178 & Transcription factor SII & $\mathrm{OL}$ & 33 & 98 & $8.00 \mathrm{E}-12$ \\
\hline DSLPV1_195 & DNA topoisomerase II & OT & 43 & 100 & 0 \\
\hline
\end{tabular}

OL Ostreococcus lucimarinus CCE9901, OT Ostreococcus tauri, MP Micromonas pusilla CCMP1545, Ms. Micromonas sp. RCC299 


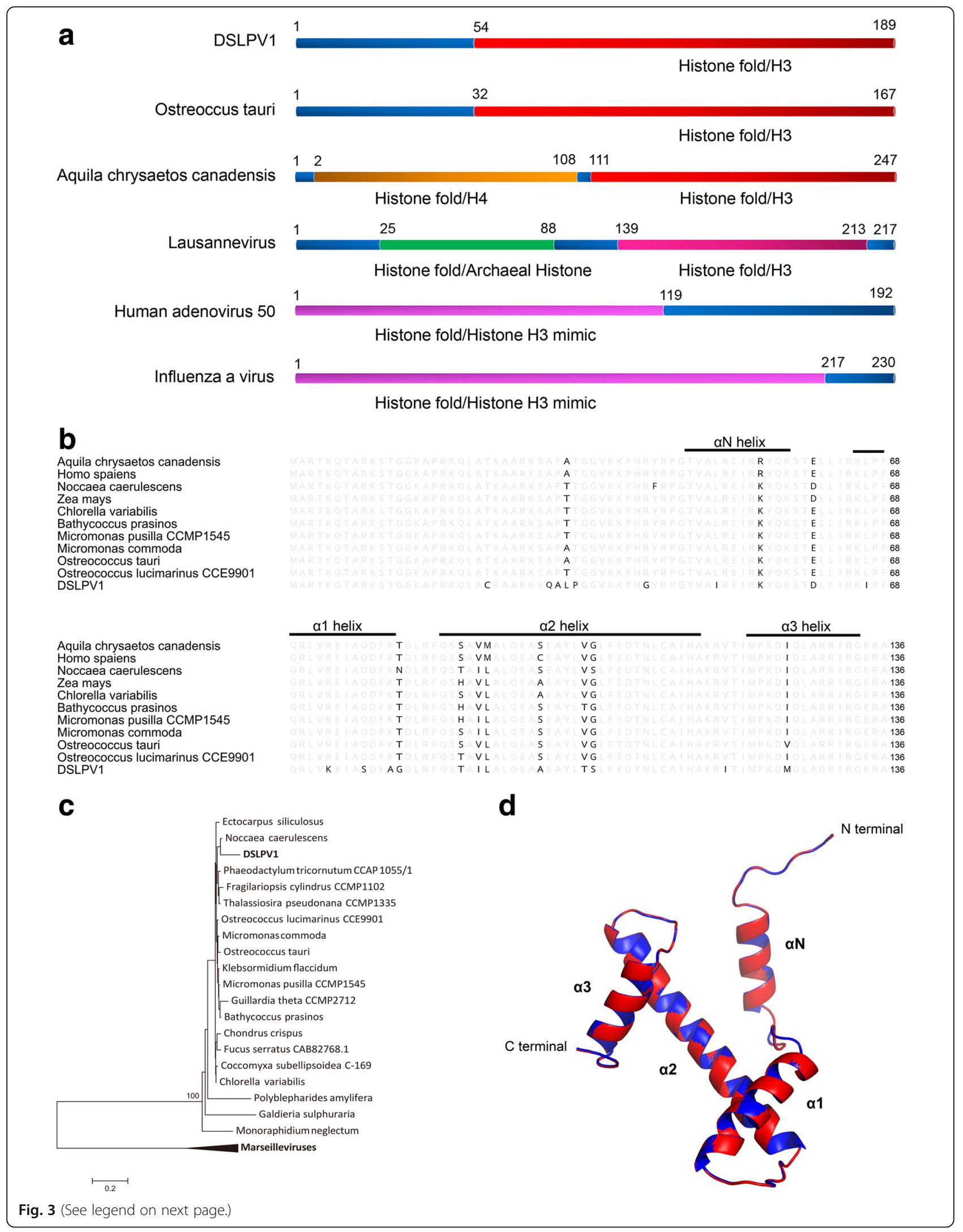


(See figure on previous page.)

Fig. 3 Viral histone H3 gene in DSLPV1. a Schematic of viral histone H3 in DSLPV1 and its relatives in representative eukaryotes and other viruses. Different histone fold regions are marked in different colors, and the similar histones are indicated in the same color, i.e., eukaryotes derived histones in red, viral histones in pink and histone mimics in purple. $\mathbf{b}$ Amino acid sequence alignments of the DSLPV1 histone fold H3 and the representative eukaryotic histone $\mathrm{H} 3$ folds. Diverged amino acids are emphatically visualized and canonical helixes of histone fold are indicated. c Maximum likelihood phylogenetic tree of the viral and the representative eukaryotic histone fold $\mathrm{H} 3$ proteins. GenBank accession numbers of the histone sequences are listed in Additional file 1: Table S4. Only more than $50 \%$ of bootstrap value is shown in the tree. $\mathbf{d}$ Predicted three dimensional structure of the histone H3 fold in DSLPV1. The protein template used for the prediction is shown in blue and the target protein is in red

YSLPVs as well as with the members in the Chlorovirus genus that are presently the only well-defined freshwater algae viruses in Phycodnaviridae [27].

Viruses that are closely related to the prasinoviruses may have the Mamiellophyceae algae like host since all known prasinoviruses infect the Mamiellophyceae algae [28-30]. Meanwhile, some evidence showed the existence of freshwater algae species in Mamiellophyceae, albeit no phycodnaviruses were detected [10]. We consequently speculate that the host of DSLPV1 may be some kinds of freshwater Mamiellophyceae algae related species. Furthermore, the closest homologues of several viral genes in DSLPV1, especially the histone $\mathrm{H} 3$ gene, are present in the Ostreococcus algae as well, e.g., O. lucimarinus, O. tauri (Table 3). These genetic links between DSLPV1 and green algae not only further support the above proposed hypothesis, but also give better insight into a theoretical guidance for the isolation of potential algal host of DSLPV1. In addition, the Mamiellophyceae related contigs are the most abundant in the algae-related contigs that were identified in the DSL metagenomic datasets (data not shown). Hence, all these evidence indicates the possibility of the interaction relationship between DSLPV1 and the Ostreococcus related algae in freshwater. To further shed insight on the host of DSLPV1, both the DSLPV1 and its algae host need to be isolated, and infection experiments had to be performed. Notably, on the phylogenetic tree of the histone fold H3 protein (Fig. 3c), DSLPV1 was grouped together with the Noccaea caerulescens plant but not with the Ostreococcus algae. However, given that the bootstrap value is less than 50\%, at almost all the branching points in the sub-tree comprising DSLPV1, the Ostreococcus algae and other representative eukaryotes, due to the high conservation of the histone fold H3 proteins, the phylogenic affiliation of the DSLPV1 histone fold H3 protein remains uncertain. Accordingly, the horizontal transfer of the histone $\mathrm{H} 3$ fold protein gene may occur between DSLPV1 and the Ostreococcus algae. It hints again the Ostreococcus related algae might be the host of DSLPV1.

Surprisingly, a eukaryote derived histone like gene was identified in the genome of DSLPV1, which is firstly reported in the phycodnaviruses. It has been suggested that histones that associate to form the nucleosome and wrap the DNA in eukaryotic cells were probably acquired or mocked by viruses [31, 32]. Viral histones carry largely unknown functions [31-38]. Significant roles that are played by a few viral histones were demonstrated. The histone $\mathrm{H} 4$ protein in insect bracoviruses that shares high sequence identity with its host gene plays a critical role in suppressing host immune responses during infection by competing with endogenous cellular H4 for incorporation into the chromatin [39]. Interestingly, the nonstructural protein 1 (NS1 protein) that is encoded by some influenza virus strains, mimicry of the histone H3, suppresses the antiviral response of host by sequestering crucial factors required for transcriptional elongation [31]. Similarly, human adenovirus protein VII mimics cellular histone $\mathrm{H} 3$ for binding host nucleosomes to sequester immune danger signals to evade immune system during infection [40]. Alike the histone $\mathrm{H} 4$ in bracoviruses, the DSLPV1 histone $\mathrm{H} 3$ showed high amino acid similarity (87\%) to the histone gene in its potential algae host except for an extra 53-aa tail at $\mathrm{N}$ terminal. In addition, sharing the same modification sites in N-terminal tail and canonical helixes in core fold between DSLPV1 and the cellular histone H3 renders us to speculate the similar functions of the DSLPV1 histone. Regretfully, the host of DSLPV1 is still unknown which debilitates the study of virus-host interaction to figure out the roles of viral histone in DSLPV1.

In our previous study, the genomes of algal large novel viruses YSLPVs and Yellowstone Lake giant virus (YSLGV) were discovered in Yellowstone Lake [14]. Meanwhile, interestingly the genomes of virophage YSLVs that are considered as the giant viral parasites were found in the same lake [16, 41]. Additionally, the genomes of virophages (Organic Lake Virophage, OLV, and Qinghai Lake Virophage, QLV) and their potential giant viral hosts, e.g., mimiviruses or phycodnaviruses, were obtained in Antarctic Organic Lake and Qinghai Lake, China [6, 42]. Coincidently, in addition to DSLPV1, a novel virophage DSLV1 that is closely related to Yellowstone Lake Virophage 3 (YSLV3) in Yellowstone Lake was discovered in Dishui Lake as well [15]. Interestingly, DSLPV1 and DSLV1 share the collagen triple helix repeat containing protein that was 
suspected to be involved in protein-protein interaction in virophage Sputnik/host mamavirus and virophage OLV/potential host Organic Lake Phycodnavirus (OLPV) associations $[6,43]$. Genetic links were also observed between the YSLPVs and the YSLVs. However, their potential associations await future exhaustively experimental study.

\section{Conclusions}

In conclusion, a complete linear genomic sequence of DSLPV1 is discovered based on sequence assembly of the metagenomic datasets from Dishui Lake in Shanghai, China. Comprehensive genomic and phylogenetic analyses reveal that DSLPV1 represents a novel viral species in freshwater aquatic ecosystem and is closely related to the marine algae infecting Prasinovirus in the family Phycodnaviridae. Recent horizontal gene transfer was detected between DSLPV1 and its potential algal host. Our results here and the previous [14] suggest that the diversity and distribution of freshwater algal large or giant viruses remain far beyond exploration. Such knowledge will significantly contribute to better understanding not only the evolution of the phycodnaviruses and other related giant viruses, e.g., mimiviruses, but also their parasite viruses of virophages.

\section{Additional file}

Additional file 1: Supplementary data. It contains all supplementary figures and tables. (DOCX $447 \mathrm{~kb}$ )

\begin{abstract}
Abbreviations
dsDNA: Double-stranded DNA; DSL: Dishui Lake; DSLPV1: Dishui Lake Phycodnavirus 1; DSLV1: Dishui Lake Virophage 1; NCLDVs: Nucleo-cytoplasmic large DNA viruses; NCVOG: Nucleo-Cytoplasmic Virus Orthologous Group; nr database: Non-redundant database; NS1 protein: Non-Structural protein 1; OLPV: Organic Lake Phycodnavirus; OLV: Organic Lake Virophage; ORF: Open Reading Frame; PCR: Polymerase Chain Reaction; QLV: Qinghai Lake Virophage; rnr2: ribonucleoside-diphosphate reductase small subunit; YSLGV: Yellowstone Lake giant virus; YSLPVs: Yellowstone Lake Phycodnaviruses; YSLV: Yellowstone Lake Virophage
\end{abstract}

\section{Acknowledgements}

We would like to thank Chaowen Gong, Yongxin Yu, Hongming Wang and Linghao Hu for their kindly help on the assembly methodology, phylogenetic analyses and image preparation. We would also like to thank Taigang Liu for supporting on BioLinux servers.

\section{Funding}

This work was supported by the National Natural Science Foundation of China (31570112, 41376135).

\section{Availability of data and materials}

The complete DSLPV1 genome sequence has been deposited in GenBank (accession no. KY747489). The phylogenetic trees (Figs. 2b and $3 \mathrm{c}$ and Additional file 1: Figure S2) have been deposited in TreeBase (http://purl.org/ phylo/treebase/phylows/study/TB2:S22094). The datasets used and/or analyzed in the current study are available from the corresponding author on reasonable request.

\section{Authors' contributions}

YW conceived and designed this study. $\mathrm{HC}$ and WZ assembled the sequences and analyzed the data. $\mathrm{HC}$ performed the experiments. $\mathrm{XL}$ performed the sampling and sequence analysis. SY and YP analyzed the data and modified the manuscript critically. HC, WZ and YW wrote the manuscript. YW analyzed the data. All authors read and approved the final manuscript.

\section{Ethics approval and consent to participate}

The water samples were collected from Dishui Lake with no permissions since it is freely available to the citizens.

\section{Consent for publication}

Not applicable.

\section{Competing interests}

The authors declare that they have no competing interests.

\section{Publisher's Note}

Springer Nature remains neutral with regard to jurisdictional claims in published maps and institutional affiliations.

\section{Author details}

${ }^{1}$ College of Food Science and Technology, Shanghai Ocean University, Shanghai, China. ${ }^{2}$ Laboratory for Marine Biology and Biotechnology, Qingdao National Laboratory for Marine Science and Technology, Qingdao, China. ${ }^{3}$ Laboratory of Quality and Safety Risk Assessment for Aquatic Products on Storage and Preservation, Ministry of Agriculture, Shanghai, China. ${ }^{4}$ Institute of Biochemistry and Molecular Cell Biology, University of Göttingen, Göttingen, Germany. ${ }^{5}$ Present address: Archaea Center, Department of Biology, Copenhagen University, DK2000 Copenhagen, Denmark.

Received: 29 November 2017 Accepted: 3 January 2018 Published online: 15 January 2018

References

1. Wilson WH, Van Etten JL, Schroeder DC, Nagasaki K, Brussaard C, Bratbak G and Suttle C. Family Phycodnaviridae. In: King AMQ, Adams MJ, Carstens EB, Lefkowitz EJ, editors.Virus taxonomy: Ninth report of the International Committee on Taxonomy of Viruses. Amsterdam: Elsevier Academic Press; 2012. p. 249-62

2. Colson P, De Lamballerie X, Fournous G, Raoult D. Reclassification of giant viruses composing a fourth domain of life in the new order Megavirales. Intervirology. 2012;55(5):321-32.

3. Iyer LM, Balaji S, Koonin EV, Aravind L. Evolutionary genomics of nucleo-cytoplasmic large DNA viruses. Virus Res. 2006;117(1):156-84.

4. Bratbak G, Heldal M, Norland S, Thingstad TF. Viruses as partners in spring bloom microbial trophodynamics. Appl Environ Microbiol. 1990;56(5):1400-5.

5. Martínez JM, Schroeder DC, Larsen A, Bratbak G, Wilson WH. Molecular dynamics of Emiliania huxleyi and cooccurring viruses during two separate mesocosm studies. Appl Environ Microbiol. 2007;73(2):554-62.

6. Yau S, Lauro FM, DeMaere MZ, Brown MV, Thomas T, Raftery MJ, Andrews-Pfannkoch C, Lewis M, Hoffman JM, Gibson JA. Virophage control of antarctic algal host-virus dynamics. Proc Natl Acad Sci. 2011;108(15):6163-8.

7. Dunigan DD, Fitzgerald LA, Van Etten JL. Phycodnaviruses: a peek at genetic diversity. Virus Res. 2006;117(1):119-32.

8. Wilson WH, Van Etten JL, Allen MJ. The Phycodnaviridae: the story of how tiny giants rule the world. Curr Top Microbiol Immunol. 2009;328:1-42.

9. Worden AZ, Nolan JK, Palenik B. Assessing the dynamics and ecology of marine picophytoplankton: the importance of the eukaryotic component. Limnol Oceanogr. 2004;49(1):168-79.

10. Marin B, Melkonian M. Molecular phylogeny and classification of the Mamiellophyceae class. Nov. (Chlorophyta) based on sequence comparisons of the nuclear- and plastid-encoded rRNA Operons. Protist. 2010;161(2):304-36.

11. Clerissi C, Grimsley N, Subirana L, Maria E, Oriol L, Ogata H, Moreau H, Desdevises $Y$. Prasinovirus distribution in the Northwest Mediterranean Sea is affected by the environment and particularly by phosphate availability. Virology. 2014;466-467:146-57.

12. Derelle E, Ferraz C, Escande ML, Eychenie S, Cooke R, Piganeau G, Desdevises Y, Bellec L, Moreau H, Grimsley N. Life-cycle and genome of OtV5, a large DNA virus of the pelagic marine unicellular green alga Ostreococcus tauri. PLoS One. 2008;3(5):e2250. 
13. Moreau H, Piganeau G, Desdevises Y, Cooke R, Derelle E, Grimsley N. Marine prasinovirus genomes show low evolutionary divergence and acquisition of protein metabolism genes by horizontal gene transfer. J Virol. 2010;84(24):12555-63.

14. Zhang W, Zhou J, Liu T, Yu Y, Pan Y, Yan S, Wang Y. Four novel algal virus genomes discovered from Yellowstone Lake metagenomes. Sci Rep. 2015:5:15131.

15. Gong C, Zhang W, Zhou X, Wang H, Sun G, Xiao J, Pan Y, Yan S, Wang Y. Novel virophages discovered in a freshwater lake in China. Front Microbiol. 2016;7:5.

16. Zhou J, Zhang W, Yan S, Xiao J, Zhang Y, Li B, Pan Y, Wang Y. Diversity of virophages in metagenomic datasets. J Virol. 2013;87(8):4225-36.

17. Quevillon E, Silventoinen V, Pillai S, Harte N, Mulder N, Apweiler R, Lopez R. InterProScan: protein domains identifier. Nucleic Acids Res. 2005;33(suppl 2):W116-20.

18. Söding J, Biegert A, Lupas AN. The HHpred interactive server for protein homology detection and structure prediction. Nucleic Acids Res. 2005;33(suppl 2):W244-8.

19. Lowe TM, Eddy SR. tRNAscan-SE: a program for improved detection of transfer RNA genes in genomic sequence. Nucleic Acids Res. 1997;25(5):955-64.

20. Kurtz S, Choudhuri JV, Ohlebusch E, Schleiermacher C, Stoye J, Giegerich R. REPuter: the manifold applications of repeat analysis on a genomic scale. Nucleic Acids Res. 2001;29(22):4633-42.

21. Darling AC, Mau B, Blattner FR, Perna NT. Mauve: multiple alignment of conserved genomic sequence with rearrangements. Genome Res. 2004;14(7):1394-403.

22. Kumar S, Stecher G, Tamura K. MEGA7: molecular evolutionary genetics analysis version 7.0 for bigger datasets. Mol Biol Evol. 2016;33(7):1870-4.

23. DeLano WL. Pymol: an open-source molecular graphics tool. CCP4 Newsl Protein Crystallogr. 2002;40:82-92.

24. Yutin N, Wolf YI, Raoult D, Koonin EV. Eukaryotic large nucleo-cytoplasmic DNA viruses: clusters of orthologous genes and reconstruction of viral genome evolution. Virol J. 2009;6(1):1

25. Rensing SA, Lang D, Zimmer AD, Terry A, Salamov A, Shapiro H, Nishiyama T, Perroud PF, Lindquist EA, Kamisugi $Y$. The Physcomitrella genome reveals evolutionary insights into the conquest of land by plants. Science. 2008:319(5859):64-9.

26. Worden AZ, Lee JH, Mock T, Rouzé P, Simmons MP, Aerts AL, Allen AE, Cuvelier ML, Derelle E, Everett MV. Green evolution and dynamic adaptations revealed by genomes of the marine picoeukaryotes Micromonas. Science. 2009;324(5924):268-72.

27. Van Etten $J$, Dunigan DD. Chloroviruses: not your everyday plant virus. Trends Plant Sci. 2012;17(1):1-8.

28. Bellec L, Grimsley N, Moreau H, Desdevises Y. Phylogenetic analysis of new Prasinoviruses (Phycodnaviridae) that infect the green unicellular algae Ostreococcus, Bathycoccus and Micromonas. Environ Microbiol Rep. 2009;1(2):114-23.

29. Clerissi C, Desdevises Y, Grimsley N. Prasinoviruses of the marine green alga Ostreococcus tauri are mainly species specific. J Virol. 2012;86(8):4611-9.

30. Derelle E, Monier A, Cooke R, Worden AZ, Grimsley NH, Moreau H. Diversity of viruses infecting the green microalga Ostreococcus lucimarinus. J Virol. 2015:89(11):5812-21.

31. Marazzi I, Ho JS, Kim J, Manicassamy B, Dewell S, Albrecht RA, Seibert CW, Schaefer $U$, Jeffrey KL, Prinjha RK. Suppression of the antiviral response by an influenza histone mimic. Nature. 2012;483(7390):428-33.

32. Thomas V, Bertelli C, Collyn F, Casson N, Telenti A, Goesmann A, Croxatto A, Greub G. Lausannevirus, a giant amoebal virus encoding histone doublets. Environ Microbiol. 2011;13(6):1454-66.

33. Cheng C-H, Liu S-M, Chow T-Y, Hsiao Y-Y, Wang D-P, Huang J-J, Chen H-H Analysis of the complete genome sequence of the $\mathrm{Hz}-1$ virus suggests that it is related to members of the Baculoviridae. J Virol. 2002;76(18):9024-34.

34. Piégu B, Guizard S, Spears T, Cruaud C, Couloux A, Bideshi DK, Federici BA, Bigot Y. Complete genome sequence of invertebrate iridescent virus 22 isolated from a blackfly larva. J Gen Virol. 2013;94(9):2112-6.

35. Piégu B, Guizard S, Spears T, Cruaud C, Couloux A, Bideshi DK, Federici BA, Bigot Y. Complete genome sequence of invertebrate iridovirus IIV-25 isolated from a blackfly larva. Arch Virol. 2014;159(5):1181-5.

36. Piégu B, Guizard S, Yeping T, Cruaud C, Asgari S, Bideshi DK, Federici BA, Bigot $Y$. Genome sequence of a crustacean iridovirus, IIV31, isolated from the pill bug, Armadillidium vulgare. J Gen Virol. 2014;95(7):1585-90.
37. de Souza RF, lyer LM, Aravind L. Diversity and evolution of chromatin proteins encoded by DNA viruses. Biochim Biophys Acta. 2010;1799(3-4):302-18.

38. Yáñez RJ, Rodríguez JM, Nogal ML, Yuste L, Enríquez C, Rodriguez JF, Viñuela E. Analysis of the complete nucleotide sequence of African swine fever virus. Virology. 1995;208(1):249-78.

39. Gad W, Kim Y. A viral histone $\mathrm{H} 4$ encoded by Cotesia plutellae bracovirus inhibits haemocyte-spreading behaviour of the diamondback moth, Plutella xylostella. J Gen Virol. 2008;89(4):931-8.

40. Avgousti DC, Herrmann C, Kulej K, Pancholi NJ, Sekulic N, Petrescu J, Molden RC, Blumenthal D, Paris AJ, Reyes ED, et al. A core viral protein binds host nucleosomes to sequester immune danger signals. Nature. 2016:535(7610):173-7.

41. Zhou J, Sun D, Childers A, McDermott TR, Wang Y, Liles MR. Three nove virophage genomes discovered from Yellowstone Lake metagenomes. J Virol. 2015;89(2):1278-85.

42. Oh S, Yoo D, Liu W-T. Metagenomics reveals a novel Virophage population in a Tibetan Mountain Lake. Microbes Environ. 2016;31(2):173-7.

43. La Scola B, Desnues C, Pagnier I, Robert C, Barrassi L, Fournous G, Merchat M, Suzan-Monti M, Forterre $P$, Koonin E. The virophage as a unique parasite of the giant mimivirus. Nature. 2008;455(7209):100-4

\section{Submit your next manuscript to BioMed Central and we will help you at every step:}

- We accept pre-submission inquiries

- Our selector tool helps you to find the most relevant journal

- We provide round the clock customer support

- Convenient online submission

- Thorough peer review

- Inclusion in PubMed and all major indexing services

- Maximum visibility for your research

Submit your manuscript at www.biomedcentral.com/submit
C Biomed Central 